\title{
Symmetries and the Polarized Optical Spectra of Exciton Complexes in Quantum Dots
}

\author{
M A Dupertuis, Fredrik Karlsson, D Y Oberli, E Pelucchi, A Rudra, \\ Per-Olof Holtz and E Kapon
}

\section{Linköping University Post Print}

N.B.: When citing this work, cite the original article.

Original Publication:

M A Dupertuis, Fredrik Karlsson, D Y Oberli, E Pelucchi, A Rudra, Per-Olof Holtz and E Kapon, Symmetries and the Polarized Optical Spectra of Exciton Complexes in Quantum Dots, 2011, Physical Review Letters, (107), 12, 127403.

http://dx.doi.org/10.1103/PhysRevLett.107.127403

Copyright: American Physical Society http://www.aps.org/

Postprint available at: Linköping University Electronic Press

http://urn.kb.se/resolve?urn=urn:nbn:se:liu:diva-71219 


\title{
Symmetries and the Polarized Optical Spectra of Exciton Complexes in Quantum Dots
}

\author{
M. A. Dupertuis, ${ }^{1}$ K. F. Karlsson, ${ }^{1,2}$ D. Y. Oberli, ${ }^{1}$ E. Pelucchi, ${ }^{1, *}$ A. Rudra, ${ }^{1}$ P. O. Holtz, ${ }^{2}$ and E. Kapon ${ }^{1}$ \\ ${ }^{1}$ Ecole Polytechnique Fédérale de Lausanne (EPFL), Laboratory of Physics of Nanostructures, CH-1015 Lausanne, Switzerland \\ ${ }^{2}$ Linköping University, Department of Physics, Chemistry, and Biology (IFM), Semiconductor Materials, S-58183 Linköping, Sweden
} (Received 8 April 2011; published 16 September 2011)

\begin{abstract}
A systematic and simple theoretical approach is proposed to analyze true degeneracies and polarized decay patterns of exciton complexes in semiconductor quantum dots. The results provide reliable spectral signatures for efficient symmetry characterization, and predict original features for low $C_{2 v}$ and high $C_{3 v}$ symmetries. Excellent agreement with single quantum dot spectroscopy of real pyramidal InGaAs/AlGaAs quantum dots grown along [111] is demonstrated. The high sensitivity of biexciton quantum states to exact high symmetry can be turned into an efficient uninvasive postgrowth selection procedure for quantum entanglement applications.
\end{abstract}

DOI: 10.1103/PhysRevLett.107.127403

PACS numbers: 78.67.Hc, 71.70.Gm, 73.21.La

Symmetries play a key role for understanding the electronic band structure of crystals [1], the optical spectra of atoms [2], or the optical properties of semiconductors [3]. Excitons are elementary excitations in semiconductors [4] and in semiconductor quantum nanostructures. An exciton is generated when an electron from the valence band is promoted to the conduction band by the absorption of a photon, thereby creating a hole in the valence band. The quantum states of the correlated electron-hole pair, the exciton, are determined by the interplay between the Coulomb interaction between the two charge carriers and the symmetries of the band edges in the crystalline solid in general [5], and of the nanostructure's shape, size and composition in particular [6]. The fine structure of an exciton confined to a quantum dot (QD) is determined by the electron-hole exchange interaction and it has been intensively studied in numerous QD structures [7,8], highlighting the influence of strain and shape [9] and the effect of charging $[10,11]$. The excitonic fine structure and the polarization of the optical transitions have profound relations with the underlying symmetries of the nanostructures. However, despite the usual interpretation of polarization anisotropy in terms of valence-band mixing [12], and a recent demonstration of the vanishing fine-structure splitting in QDs [13,14], a general understanding of the relation between symmetry and the complex polarization spectra of excitons and excitonic complexes is still lacking.

We shall first show in this Letter that the present understanding of the polarization properties of excitons in strongly confined $C_{2 v}$ QDs-a common widespread symmetry-is in drastic contrast with general grouptheoretical considerations. Next we present our approach, which makes simultaneous use of basic qualitative information available on the first few QD electron and hole states. We show that new light can be shed on degeneracy lifting, on the nature of dark states, on polarized decay of excitons $(X)$ and biexcitons $(2 X)$, even in more complicated $C_{3 v}$ symmetry QDs. Detailed analysis of polarized photoluminescence (PL) of pyramidal QDs, fully establishes the power of this approach.

$C_{2 v}$ QDs have been intensively investigated since they are produced readily in Stranski-Krastanov growth mode [7]. A prominent $C_{2 v}$ feature is the fine-structure splitting between the $x$ - and $y$-polarized bright exciton states, induced by the exchange interaction [7]. Another wellknown feature is the alleged presence of two dark states with parallel spins [15]; this widespread description is however in contradiction with a simple group-theoretical study of such excitons.

A group-theoretical approach of the polarization properties of the excitonic states in QDs requires three steps: (i) identification of the $\mathrm{QD}$ point group $(\mathrm{PG})$, resulting from the common symmetry elements between the crystal symmetry and the QD symmetry (mesoscopic level), (ii) labeling each quantum state of interest with its global symmetry properties, i.e., with irreducible representations $\Gamma$ (irreps) of the PG, (iii) the use of optical selection rules, given by the Wigner-Eckart theorem.

For $C_{2 v}$ QDs, the irreps labeling the symmetry of the ground electron and hole states $e_{1}$ and $h_{1}$ may only be $\Gamma_{e_{1}}$, $\Gamma_{h_{1}}=E_{1 / 2}$, since there is only one double group irrep in $C_{2 v}$. This holds independently of any model. Then one can immediately determine the symmetry labels of the excitonic product states of $e_{1}$ and $h_{1}$ using the multiplication tables [16]: $E_{1 / 2} \times E_{1 / 2}=A_{1}+B_{1}+B_{2}+A_{2}$. Assuming the strong confinement limit (SCL), Coulomb interactions will slightly lift their degeneracy within a configuration [17]. It follows that in strongly confined $C_{2 v}$ QDs there exist only four kinds of ground states of $X$ to which one should attach the labels $A_{1}, B_{1}, B_{2}$ and $A_{2}$. To know their optical activity, we recall that the dipole moment $\mu_{k}$, $k=x, y, z$ transforms like vectors along $x, y, z$, which are labeled with irreps $B_{1}, B_{2}, A_{1}$, respectively, (convention of [16]). Consequently using the Wigner-Eckart theorem we find that each of the three states labeled $A_{1}, B_{1}, B_{2}$ is optically active in a specific linear polarization, while there 
exists only one dark state labeled $A_{2}$. These general results are in sharp contrast with current understanding, for which two dark states exist $[15,18]$. It is possible to show [17] that the nondegenerate character of states $A_{1}$ and $A_{2}$ can be interpreted as a sign of valence-band mixing, an effect akin to III-V QDs. The optical activity in the $z$ direction of the state $A_{1}$ can consistently be attributed to mixing of the ground heavy hole $(\mathrm{HH})$ state with some light hole $(\mathrm{LH})$ component. An approach to polarization anisotropy solely based on valence-band mixing arguments is, however, unable to match the strict group-theoretical prediction that every state couples uniquely to its own single linear polarization, and that there is only one state remaining strictly dark.

For higher symmetry, there are more double group irrep labels. We consider now $C_{3 v}$ QDs, like pyramidal zinc blende QDs grown in the [111] direction [14]. HH and LH now refer to Bloch functions labeled $u_{h, j=(3 / 2), m}$, where $m$ is associated with the angular momentum $J_{z}$ along [111]. One must associate global symmetry labels to $e_{1}$, $h_{1}$, and $h_{2} \cdot e_{1}$ is necessarily labeled with irrep $E_{1 / 2}$ of $C_{3 v}$. As $h_{1}$ and $h_{2}$ dominantly display ground $\mathrm{HH}$ and ground LH character [19], in agreement with their oblate or prolate spheroidal shapes ( $h_{2}$ hybridizes largely with the connected vertical quantum wire), one can associate irreps $E_{3 / 2}$ (strictly speaking ${ }^{1} E_{3 / 2}+{ }^{2} E_{3 / 2}$ ) and $E_{1 / 2}$ to $h_{1}$ and $h_{2}$, respectively. This can be done by considering the dominant contribution to every wave function as being a product of a single envelope function and a heterostructure-symmetrized hole Bloch function [20] (the latter can also be considered as a simple "discrete PG pseudospin" (DPGPS) [17]). For example, the symmetry of a ground LH-like state must be the same as in the product $\phi_{L H}^{A_{1}}(\mathbf{r}) u_{L H}^{E_{1 / 2}, \gamma}(\mathbf{r})$, where $\phi_{L H}^{A_{1}}$ is the envelope and $u_{L H}^{E_{1 / 2}, \gamma}=u_{h,(3 / 2), m}, \gamma=\frac{3}{2}-m, m= \pm \frac{1}{2}$ is the DPGPS $(\gamma$ is the partner function index linked with irrep $E_{1 / 2}$ [16]).
Note that $u_{h, 3 / 2), m}$ is a hole Bloch function, i.e., the proper time conjugate of its valence-band electron image. Previous theoretical and experimental work [21,22] has shown that several subtle features of $C_{3 v}$ quantum wires and QDs could be interpreted if one assumes an additional symmetry plane $\sigma_{h}$ perpendicular to [111], leading to an effective $D_{3 h}$ PG, an effect called "symmetry elevation." Intuitively, one may justify this: (i) the crystalline bulk structure of GaAs displays in many respects only weak inversion symmetry breaking, (ii) on the mesoscopic side, a pyramidal $C_{3 v}$ QD may be considered as a weakly curved (with respect to $\sigma_{h}$ ) oblate spheroid for $\mathrm{HH}-$ like $h_{1}$, and as a weakly deformed prolate spheroid for LH-like $h_{2}$, as demonstrated by eight-band $k \cdot p$ calculations [22]. In this case we assign the labels $E_{3 / 2}$ and $E_{5 / 2}$ to $h_{1}$ and $h_{2}$ respectively, if one keeps the label $E_{1 / 2}$ for $e_{1}$. These symmetry assignments, if correct, will no longer depend on a particular description $(k \cdot p$ model, pseudopotential approach, etc.) as they only refer to basic global transformation properties of quantum states $e_{1}, h_{1}$ and $h_{2}$. They will be enough for building the lower lying complexes in the SCL.

We now assign the symmetry to lower lying groups of $X$ and $2 X$ states in the SCL by again using the product rule. In $C_{3 v}$, the HH-like exciton states issuing from $h_{1}$ (denoted here $\left.X_{10}\right)$ are described by $E_{1 / 2} \times E_{3 / 2}=E+E$, while one finds $E^{\prime}+E^{\prime \prime}$ in $D_{3 h}$. The LH-like exciton states (denoted $X_{01}$ ) are described by the product $E_{1 / 2} \times E_{1 / 2}=$ $A_{1}+E+A_{2}$ in $C_{3 v}$ or $A_{1}^{\prime}+E^{\prime \prime}+A_{2}^{\prime}$ in $D_{3 h}$. Fundamental twofold degeneracies appear, linked with two-dimensional $E$-type irreps. For $2 X$ states, one should first make the products for electron and holes separately, to easily account for Pauli exclusion. When two electrons (holes) occupy the same $e_{1}\left(h_{1}\right)$ state, they are in a restricted configuration and globally display $A_{1}$ symmetry. As a result, the nondegenerate ground biexciton (denoted

TABLE I. Typical symmetries of the first quantum states (individual carriers and excitons) in the case of point group symmetries $C_{2 v}, C_{3 v}$ and $D_{3 h} . X_{i j}$ and $2 X_{i j}$ refers to excitons and biexcitons respectively, with all electrons in $e_{1}$, and $i, j$ holes in levels $h_{1}, h_{2}$, respectively.

\begin{tabular}{lccc}
\hline \hline$\uparrow_{x}^{z}$ & & & \\
Carrier & $C_{2 v}$ & $C_{3 v}$ & $D_{3 h}$ \\
\hline$e_{1}$ & $E_{1 / 2}$ & $E_{1 / 2}$ & $E_{1 / 2}$ \\
$h_{1}$ & $E_{1 / 2}$ & $E_{1 / 2}$ & $E_{5 / 2}$ \\
$h_{2}$ & $E_{1 / 2}$ & $C_{3 v}$ & $D_{3 h}$ \\
\hline \hline Complex & $C_{2 v}$ & $E+E$ & $E^{\prime}+E^{\prime \prime}$ \\
\hline$X_{10}$ & $A_{1}+B_{1}+B_{2}+A_{2}$ & $A_{1}+E+A_{2}$ & $A_{2}^{\prime \prime}+E^{\prime}+A_{1}^{\prime \prime}$ \\
$X_{01}$ & $A_{1}+B_{1}+B_{2}+A_{2}$ & $A_{1}($ Pauli restriction $)$ & $A_{1}^{\prime}($ Pauli restriction) \\
$2 X_{20}$ & $A_{1}($ Pauli restriction $)$ & $E+E$ & $E^{\prime}+E^{\prime \prime}$ \\
$2 X_{11}$ & $A_{1}+B_{1}+B_{2}+A_{2}$ & ${ }^{2}$ &
\end{tabular}



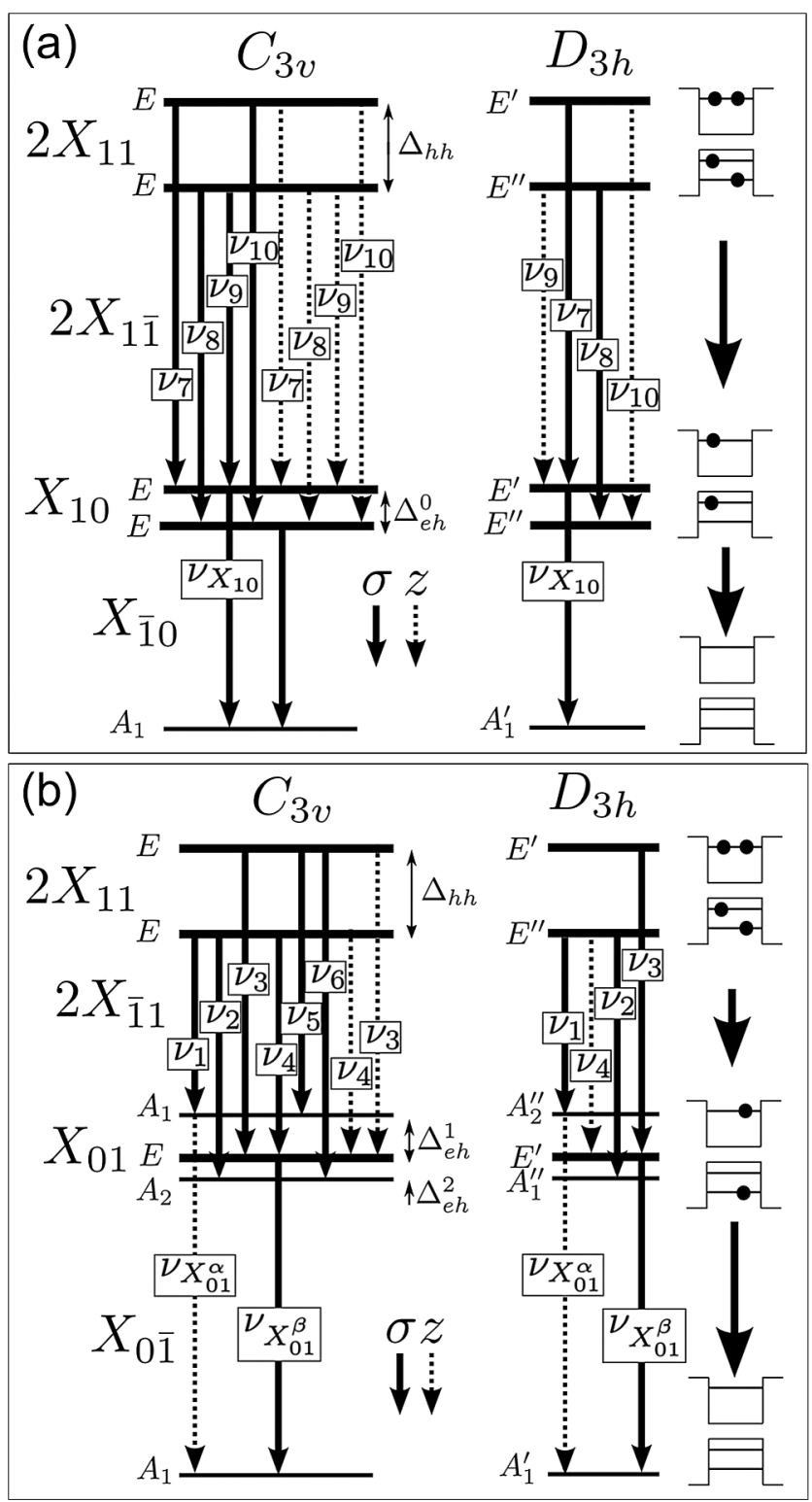

FIG. 1. Predicted polarized radiative decay paths of $2 X_{11}$. Solid and dotted lines represent allowed transitions with $\sigma$ and $z$ polarization, respectively, $\left(\sigma \equiv x / y / \sigma_{ \pm}\right)$.

$2 X_{20}$ ) also bear the label $A_{1}$ (or $A_{1}^{\prime}$ in $D_{3 h}$ ). Furthermore the biexciton with two electrons in $e_{1}$ and two holes in configuration $\left(h_{1}, h_{2}\right)$ (denoted $\left.2 X_{11}\right)$ will give rise to four states since $\left({ }^{1} E_{3 / 2}+{ }^{2} E_{3 / 2}\right) \times E_{1 / 2}=E+E$ in $C_{3 v}$ (and $E_{3 / 2} \times E_{1 / 2}=E^{\prime}+E^{\prime \prime}$ in $D_{3 h}$ ). Hence the $2 X_{11}$ states will be twofold degenerate and correspond to the irrep $E$ in $C_{3 v}$, or $E^{\prime}$ and $E^{\prime \prime}$ in $D_{3 h}$.

Let us now turn to the possible optical decay paths. To this end, one further needs the symmetry of the dipole moments $\mu_{k}, k=x, y, z$ (Cartesian vector components), and one finds $E$ for $(x, y)$ and $A_{1}$ for $z$, respectively, in symmetry $C_{3 v}$. In $D_{3 h}$ the corresponding result is $E^{\prime}$ for $(x, y)$ and $A_{2}^{\prime \prime}$ for $z$. To evaluate the possibility of an optical transition from the initial $2 X$ or $X$ state $\left|X_{\text {in }}\right\rangle$ to the final
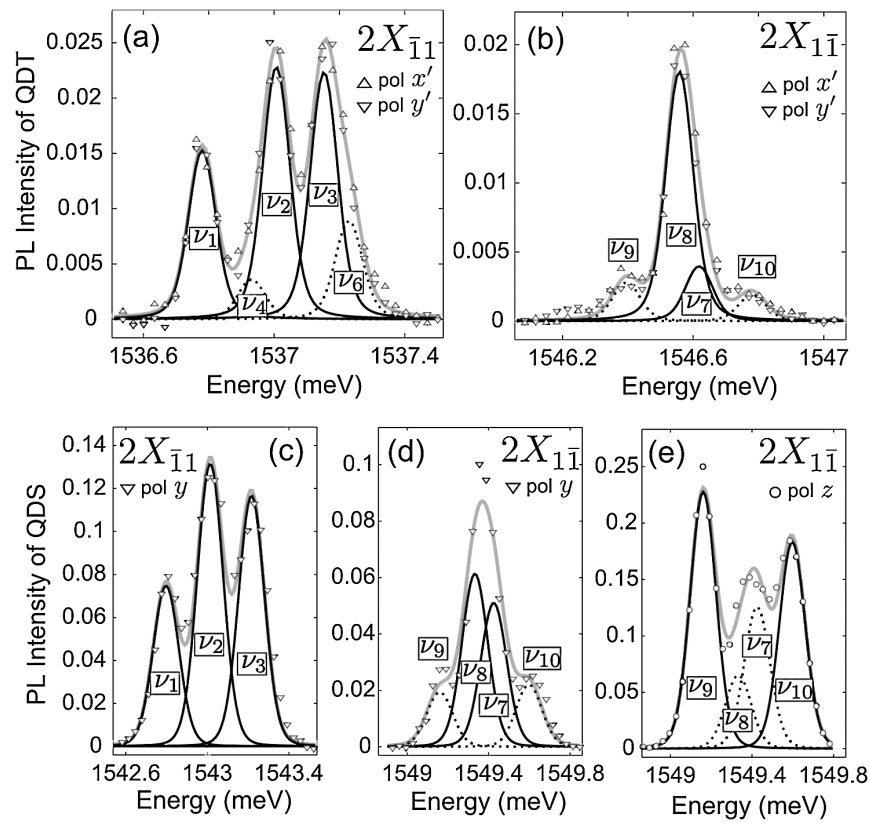

FIG. 2. Polarization resolved PL spectra of QDT (a) and (b) and QDS (c)-(e), normalized to the intensity of $X_{\overline{1} 0}$. Grey solid lines are peak fits, with the individual peaks shown below in solid (dotted) black lines for transitions allowed (forbidden) under $D_{3 h}$, for the measured polarization direction. [The fit yields vanishing intensity of $\nu_{5}$ in (a).]

state $\left|X_{\text {fin }}\right\rangle$ ( $X$ or vacuum), or to examine polarization isotropy [17], one must consider $\left\langle X_{\text {fin }}\left|\mu_{k}\right| X_{\text {in }}\right\rangle, k=$ $x, y, z$ with the Wigner-Eckart theorem. All the relevant complexes are summarized in Table I, and optical decay paths are represented in Fig. 1. A few comments are worth making: (i) the higher the symmetry, the more selective are the selection rules, remarkably symmetry elevation does not produce new degeneracies, (ii) the oscillator strength for $C_{3 v}$ (and $D_{3 h}$ ) is isotropic in the $x y$ plane, and (iii) surprisingly only one of the bright $\mathrm{HH}$-like $X_{10}$ states remains bright in $D_{3 h}$ symmetry $\left(E^{\prime}\right)$, the other becoming dark $\left(E^{\prime \prime}\right)$.

Experiments were performed on arrays of QDs fabricated by low-pressure organometallic chemical vapor deposition in inverted tetrahedral micro-pyramids patterned on a $2^{\circ}$-off GaAs (111)B substrate. Thin QDs $(\sim 1.5 \mathrm{~nm})$ selfformed due to growth anisotropy and capillarity effects from a nominally $0.5 \mathrm{~nm}$ thick $\operatorname{In}_{0.10} \mathrm{Ga}_{0.90}$ As layer at the center of the pyramids were sandwiched between $\mathrm{Al}_{0.30} \mathrm{Ga}_{0.70} \mathrm{As}$ barriers. Individual back-etched QDs were studied at a temperature of $10 \mathrm{~K}$ by means of micro-PL $(\sim 1 \mu \mathrm{m}$ spot size) with a spectral resolution of $50 \mu \mathrm{eV}$. The samples were investigated both in a top-view geometry with PL signal collected along the $z$ direction [111], and in a side-view geometry with the signal collected from the cleaved edge along the $x$ direction [1 10$]$. The linear polarization in the $x y$ plane and in the $y z$ plane could be analyzed (with a contrast of 50:1) for the two geometries by rotating a $\lambda / 2$ phase retardation plate placed 
in conjunction with a fixed linear polarizer in the signal path. In this Letter, data of two $C_{3 v}$ symmetry QDs will be presented, one measured from the top (QDT) and the other from the side (QDS).

The attention is here restricted to the excited biexciton $2 X_{11}$ and to the two single-excitons $X_{10}$ and $X_{01}$. The measured biexciton optical transitions $2 X_{\overline{1} 1}$ and $2 X_{1 \overline{1}}$ (denoted by a bar above the index of the recombining hole) are presented in Fig. 2 and should be analyzed using the derived decay schemes in Fig. 1. Identification of the emission lines can be achieved with the aid of strict energy relations stemming from these decay schemes. For example, both cascades $2 X_{1 \overline{1}} \rightarrow X_{\overline{1} 0}$ and $2 X_{\overline{1} 1} \rightarrow X_{0 \overline{1}}$ have identical initial and final states, implying $\nu_{3}+\nu_{X_{01}^{\beta}}=$ $\nu_{7}+\nu_{X_{10}}$ and $\nu_{1}+\nu_{X_{01}^{\alpha}}=\nu_{9}+\nu_{X_{10}}$. Since the energy spacings between some emission lines are comparable with their spectral linewidths, the PL spectra are analyzed by peak fitting. The $x$ - $(y-)$ polarized spectra of QDT reveal a high degree of polarization isotropy [in Figs. 2(a) and 2(b)], as theoretically expected for $C_{3 v}$ symmetry. Accordingly, the $x y$-averaged spectra of QDT were fitted accounting for the $C_{3 v}$ transitions in Fig. 1, assuming Voigt peak profiles with identical linewidths for all $2 X_{11}$ transitions. The result shown in Fig. 2(a) demonstrates that $2 X_{\overline{1} 1}$ is dominated by three emission lines $\nu_{1-3}$, in consistency with symmetry elevation from $C_{3 v}$ to $D_{3 h}$, for which only three transitions $\nu_{1-3}$ are optically allowed out of the predicted set of six $\nu_{1-6}$. Also for $2 X_{1 \overline{1}}$, the major contribution comes from the $D_{3 h}$ transitions $\nu_{7-8}$ [see Fig. 2(b)].

The group-theoretical predictions were also verified for vertically polarized transitions; to this aim, the analysis was performed for polarizations in the $y z$ plane on QDS [see Figs. 2(c)-2(e)]. Both $\sigma$ - and $z$-polarizations were observed for $2 X_{1 \overline{1}}$, in agreement with Fig. 1(a), while only $\sigma$-polarized components could be detected for $2 X_{\overline{1} 1}$. The latter fact can be understood only by the strong $\mathrm{HH}$ like character of $h_{1}$, which strongly reduces the oscillator strength along $z$. QDS exhibits $\sim 50 \%$ wider spectral linewidth than QDT, and in this case a reliable fit with six peaks $\left(\nu_{1-6}\right)$ for $2 X_{\overline{1} 1}$ could not be obtained. The expectedly weak $\nu_{4-6}$ transitions were therefore excluded from the peak fit for QDS. The effect of symmetry elevation is again clearly observed for $2 X_{1 \overline{1}}$ in Figs. 2(d) and 2(e), where $\nu_{7-8}$ dominates the $\sigma$-polarization, while $\nu_{9-10}$ dominates the $z$ polarization, in consistency with the $D_{3 h}$ selection rules in Fig. 1(a). Possible polarization crosstalk is minor [17], and the fact that none of $\nu_{7-10}$ are completely polarized for QDT and QDS reflects the approximate nature of symmetry elevation: all four transitions are allowed for any polarization vector for the actual symmetry $C_{3 v}$ [see Fig. 1(a)].

Furthermore the fine structure of $2 X_{11}, X_{10}$ and $X_{01}$, caused by electron-hole exchange $\left(\Delta_{e h}\right)$ and hole-hole exchange $\left(\Delta_{h h}\right)$, can be fully extracted from the measured transition energies according to relations derived from Fig. 1, namely $\nu_{2}-\nu_{1}=\Delta_{e h}^{1}+\Delta_{e h}^{2}, \nu_{3}-\nu_{2}=$ $\Delta_{h h}-\Delta_{e h}^{2}$, and $\nu_{7}-\nu_{8}=\Delta_{h h}-\Delta_{e h}^{0}, \nu_{8}-\nu_{9}=\Delta_{e h}^{0}$.

The resulting experimental values of splitting energies for QDT and QDS are summarized in Table II. Note that the emission patterns of $2 X_{11}$ also provide the information about the dark states of $X_{10}$ and $X_{01}$, which are otherwise not accessible by any direct optical measurements of these excitons (or from the ground biexciton $2 X_{20}$ ). The dark state of $X_{10}$ was predicted numerically by pseudopotential calculations [13]. Nevertheless, this staggering effect is fundamentally explained only by invoking symmetry elevation (using label $E^{\prime \prime}$ of $D_{3 h}$ ). The absence of a corresponding $X_{\overline{1} 0}$ and $X_{\overline{2} 0}$ transitions is confirmed in the optical spectra supplied in [17].

It should be pointed out that any breaking of the symmetry below $C_{3 v}$ would be evidenced by lifting the degeneracy and $x y$-polarization isotropy of the $E$-type states. The $E$ levels of $X_{10}, X_{01}$, and $2 X_{11}$ were carefully verified for QDT, and a splitting of $\nu_{X_{10}}$ (into $x$ - and $y$-polarized components) could not be resolved within the precision of the measurements, $\sim 5 \mu \mathrm{eV}$. Such splittings, particularly on the biexciton lines, do form very sensitive probes of the exact $C_{3 v}\left(D_{3 h}\right)$ symmetry of the QD [17].

Finally, we emphasize that our approach is applicable to all QD systems with symmetry, whether they are nearly strain-free GaAs/AlGaAs QDs, or InGaN and AlGaN QDs with close uppermost valence bands and strain. Such variety enables different routes towards QD-based optical quantum information technologies. Our method is independent of specifics like detailed shape, strain, or valenceband mixing, and provides comprehension of the role of spin and of the excitonic fine-structure which are always of uppermost importance in this context [23].

To conclude, we propose a general approach for the understanding of the fine-structure of complexes in QDs that does not require heavy computations and provides sets of consistent spectroscopic signatures able to identify particularly symmetric quantum states. The entire predicted emission patterns of $X$ and $2 X$ are completely mapped from the experiment, including polarization dependence and the strictly dark states. We have evidenced $X$ and $2 X$ states in pyramidal QDs that indeed possess the high $C_{3 v}$ symmetry, and studied subtle effects associated with signatures of symmetry elevation towards $D_{3 h}$. Furthermore, this approach predicts features previously missed by other approaches, e.g., that $C_{2 v}$ QDs possess strictly one dark ground exciton state and not two. These results may influence the design and the choice of QDs tailored for quantum information processing.

TABLE II. Extracted exchange splittings $(\Delta)$ in units of $\mu \mathrm{eV}$.

\begin{tabular}{lllll}
\hline \hline & $\Delta_{e h}^{0}$ & $\Delta_{e h}^{1}$ & $\Delta_{e h}^{2}$ & $\Delta_{h h}$ \\
\hline QDT & 162 & 151 & 76 & 222 \\
QDS & 172 & 155 & 62 & 265 \\
\hline \hline
\end{tabular}


*Present address: Tyndall National Institute, University College Cork, Cork, Ireland.

[1] L. P. Bouckaert, R. Smoluchowski, and E. Wigner, Phys. Rev. 50, 58 (1936).

[2] E.U. Condon and H. Odabasi, Atomic structure (Cambridge University Press, Cambridge, England, 1980).

[3] J. J. Hopfield, J. Phys. Chem. Solids 15, 97 (1960).

[4] R.S. Knox, Theory of excitons, Solid State Physics (Academic Press, New York, 1963).

[5] K. Cho, Phys. Rev. B 14, 4463 (1976).

[6] G. Bester, S. Nair, and A. Zunger, Phys. Rev. B 67, 161306 (R) (2003).

[7] M. Bayer et al., Phys. Rev. Lett. 82, 1748 (1999).

[8] R. J. Warburton et al., Nature (London) 405, 926 (2000).

[9] R. Seguin et al., Phys. Rev. Lett. 95, 257402 (2005).

[10] B. Urbaszek et al., Phys. Rev. Lett. 90, 247403 (2003).

[11] M. Ediger et al., Phys. Rev. Lett. 98, 036808 (2007).

[12] T. Tanaka et al., Appl. Phys. Lett. 62, 756 (1993).
[13] R. Singh and G. Bester, Phys. Rev. Lett. 103, 063601 (2009).

[14] Mohan et al., Nat. Photon. 4, 302 (2010).

[15] E. Poem et al., Nature Phys. 6, 993 (2010).

[16] S. L. Altmann and P. Herzig, Point-Goup Theory Tables (Clarendon Press, Oxford, 1994).

[17] See Supplemental Material at http://link.aps.org/ supplemental/10.1103/PhysRevLett.107.127403 for complementary proofs related to the global symmetry approach, and for complementary experimental data.

[18] J. W. Luo et al., New J. Phys. 11, 123024 (2009).

[19] K.F. Karlsson et al., Appl. Phys. Lett. 89, 251113 (2006).

[20] S. Dalessi and M. A. Dupertuis, Phys. Rev. B 81, 125106 (2010).

[21] S. Dalessi, F. Michelini et al. (to be published).

[22] K. F. Karlsson et al., Phys. Rev. B 81, 161307 (2010).

[23] L. Xiaoqin et al., Science 301, 809 (2003); N. Akopian et al., Phys. Rev. Lett. 96, 130501 (2006). 